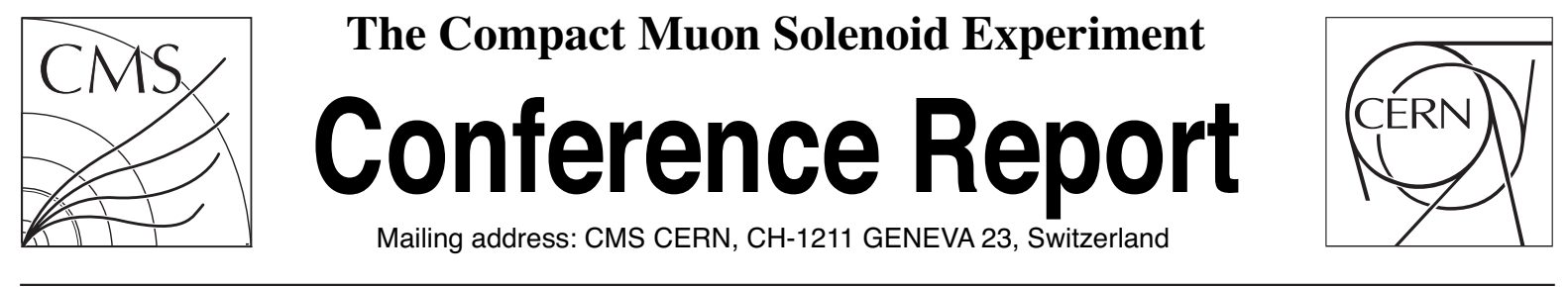

07 October 2008

\title{
CMS Tracker Alignment Strategy and First Results obtained from Cosmic Muon Tracks
}

\author{
J. Olzem
}

1. Physikalisches Institut B, RWTH Aachen University

\begin{abstract}
The all-silicon design of the CMS tracker poses new challenges in aligning the system with more than 15,000 independent modules. For optimal track-parameter resolution, the position and orientation of its modules need to be determined with a precision of better than few dozens of micrometres. Starting with the survey measurements and corrections provided by the hardware alignment system, the ultimate precision can be achieved with data from the silicon modules traversed in-situ by charged particles. Several implementations of statistical algorithms allow to solve the optimization problem with the required accuracy in manageable time. Survey measurements and experience with the hardware alignment system are described as well as the selection of data samples used for track based alignment and results from Monte-Carlo studies. First CMS Tracker alignment analysis results with cosmic track data are given.
\end{abstract}

Presented at 8th International Conference on Position Sensitive Detectors, Glasgow, United Kingdom, September 3, 2008 


\section{The CMS tracker}

The CMS silicon tracker, comprising an inner pixel and an outer strip detector, is the largest silicon detector ever built with a total active area of about $200 \mathrm{~m}^{2}$. The pixel detector consists of 1,440 modules with a total of 66 million channels and is composed of three $53 \mathrm{~cm}$ long barrel layers closed by two end cap disks of $30 \mathrm{~cm}$ diameter on either side. It is enclosed by the strip detector which is itself divided into an inner barrel/end cap structure, Tracker Inner Barrel (TIB) and Tracker Inner Disks (TID), and an outer structure consisting of an outer barrel (TOB) and two end caps (TEC). Its overall dimensions are $5.6 \mathrm{~m}$ length and $2.4 \mathrm{~m}$ diameter. A detailed description of the silicon tracker can be found in [1].

The silicon strip tracker shows a highly hierarchical structure. Its basic elements are the modules, each carrying one or two sensors and the front-end electronics. Single sided as well as double sided ("stereo") modules are used with a spatial hit resolution of 23-60 $\mu \mathrm{m}$. The TIB and TOB modules are arranged in four or six cylindrical layers, respectively, on longitudinal structures parallel to the beam (which defines the z-axis) mounted on the layer half-barrels. TID modules are grouped in three disk units per side on ring-shaped concentrical structures. In the TEC, modules are placed in ring structures on wedge shaped "petals" which are themselves attached on both sides of nine large disks staggered along $\mathrm{z}$.

\section{The overall alignment strategy}

Due to limited assembly precision, the modules of the silicon strip detector are expected to be misaligned at CMS startup, with deviations from the design positions up to the order of several hundred micrometres. In the course of the experiment, misalignment may also originate from temperature cycles during maintenance $(\Delta T \approx 30 \mathrm{~K})$, magnetic field cycles, or drying out of materials, amongst others.

For optimal track-parameter resolution, the position and orientation of the modules need to be determined with a precision of better than a few dozens of micrometres. This challenging task can only be accomplished using all available sources of data in combination with sophisticated methods of analysis. The overall alignment strategy for the CMS silicon tracker comprises a series of subsequent levels, given in the order of increasing alignment precision:

- the use of assembly knowledge and data from a wide variety of survey measurements;

- a hardware-based laser alignment system for the initial measurement and permanent fast monitoring of the large-scale support structure's geometry;

- high precision track based alignment for measuring all modules' individual spatial and rotational position parameters using redundant algorithms and various different data sets such as a wide range of collision data, beam halo and cosmic muons.

\section{Assembly knowledge and survey data}

Knowledge from survey measurements of the CMS tracker serves as an important input to the alignment procedure. They deliver module position and orientation information necessary for the convergence of high precision algorithms at startup and provide constraints on degrees of freedom which are only weakly measured with tracks.

A wide variety of data are available such as from gantry robots during tracker assembly, surveys with coordinate measurement machines (CMM) and from photogrammetry. The forward pixel detector has been extensively surveyed, yielding a resolution of $5 \mu \mathrm{m}$ in $\mathrm{z}$ and about $1 \mu \mathrm{m}$ in the $\mathrm{x}-\mathrm{y}$-plane. The TIB and TID subdetectors have been subject to CMM measurements down to single module level and global photogrammetry, while TOB and TEC were optically surveyed on half-barrel and disk level with a precision between $50 \mu \mathrm{m}$ and $150 \mu \mathrm{m}$.

\section{The laser alignment system}

The task of the hardware based laser alignment system (LAS) [2] is the initial absolute measurement of the largescale support structure of the silicon strip tracker with an accuracy below $70 \mu \mathrm{m}$, and its permanent in-time monitoring relative to a previous measurement with better than $30 \mu \mathrm{m}$ precision. The LAS uses a system of near-infrared laser beams which traverse the silicon in the tracker volume. By inducing signals on the silicon sensors, they span 
a reference system for the inter-alignment of the barrel subdetectors and the TEC disks. The inner disks and the pixel detector are not surveyed by the LAS.

The laser beams are eight-fold distributed along the $\phi$ coordinate of the tracker and divided into two groups:

- eight "alignment tube" beams range from end cap to end cap and interconnect TIB, TOB and both TEC at a radius of $56.4 \mathrm{~cm}$ from the beam line;

- two separate systems of end cap internal beams provide inter-alignment of the TEC disks. In each TEC, a total of 16 beams traverse all silicon layers at radii of $56.4 \mathrm{~cm}$ or $84.0 \mathrm{~cm}$, respectively.

By inducing signals on the silicon modules the LAS requires no additional sensors and makes use of the existing data acquisition system of the tracker. Single laser hits can be reconstructed with a spatial resolution of $30 \mu \mathrm{m}$. The LAS has a large sensitive range for residual measurements up to several millimetres; furthermore, it provides very fast response, so that full measurement and reconstruction can be performed on the time scale of a minute.

\section{Track based alignment}

The ultimate alignment precision is achieved with track based methods using data from the silicon modules traversed in-situ by charged particles. The working principle is as follows. The residuals $\mathbf{r}_{i}(\mathbf{p}, \mathbf{q})$ are defined as the displacements of hit positions with respect to the trajectory impact point of a track. They are functions of the alignment parameters $\mathbf{p}$ (i.e. the modules' positions and orientations) as well as the track parameters q. A chi-square function can be defined as

$$
\chi^{2}=\sum_{i}^{\text {tracks }} \mathbf{r}_{i}^{T}(\mathbf{p}, \mathbf{q}) \mathbf{V}_{i}^{-1} \mathbf{r}_{i}(\mathbf{p}, \mathbf{q})
$$

with the covariance matrix $\mathbf{V}_{i}$ containing the measurement uncertainties. Minimizing equation (1) provides access to the alignment parameters. The latter usually show large and complex correlations between different modules due to the hierarchical structure of the tracker.

For tracker alignment, three independent and redundant approaches are simultaneously deployed for solving the minimization problem:

- the Hits and impact points (HIP) algorithm [3] performs the analytical inversion of the $6 \times 6$ covariance matrix for individual modules. Correlations between modules are accounted for by iterative track refitting;

- the Kalman filter algorithm [4] sequentially updates the global parameter set on a track-by-track basis and keeps track of correlations with a bookkeeping facility;

- a global least square solution for $\mathbf{p}$ and $\mathbf{q}$ is calculated by the MillePede algorithm with all correlations considered. Clever algorithms in the MillePede-II program [5] avoid the inversion of the full covariance matrix.

\section{Alignment with cosmic muon tracks}

First experience with the CMS tracker alignment strategy was gained in the tracker integration facility (TIF) at CERN. About $15 \%$ of the silicon strip modules were operational during several months of cosmic muon data taking in spring and summer of 2007. Besides cosmic muon tracks, data from survey measurements and from the laser alignment system were employed in the alignment procedure.

Cosmic muon events were triggered with two groups of movable plastic scintillators above and below the detector, while a lead shield absorbed muons with momenta below $180 \mathrm{MeV} / c$. The magnetic field and the pixel detector were not present. In total, five million events have been recorded at varying temperatures of the silicon sensors ranging from $-15^{\circ} \mathrm{C}$ to $15^{\circ} \mathrm{C}$. Furthermore, tracking and alignment performance were validated using simulated events. Input data for the track based alignment were carefully selected; only events with one single muon track in a region according to the fiducial scintillator positions were considered. Tracks were required to be of good quality with a normalised $\chi^{2}$ below 4.0 and to consist of at least five well defined and isolated hits. The combined selection efficiency is about $8.3 \%$. 
Figure 1 shows the distributions of absolute $\chi^{2}$-values of the track fits assuming design geometry, geometry based on survey information, and for the geometry determined with the HIP algorithm. Compared to the design geometry, the use of both survey data and results from track based alignment significantly improve track quality, as visible in the decrease of the distributions' mean values with respect to the initial (design-) value. Furthermore, as can be seen in Figure 2 for a reference subset of modules, all three track based algorithms show consistent performance.

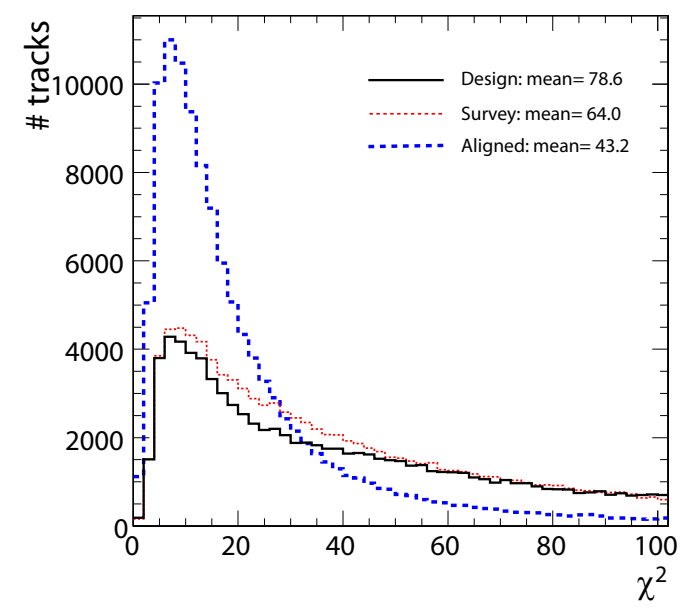

Figure 1: Distributions of the absolute $\chi^{2}$-values of the track fits for the design and survey geometries as well as the one from HIP track based alignment.

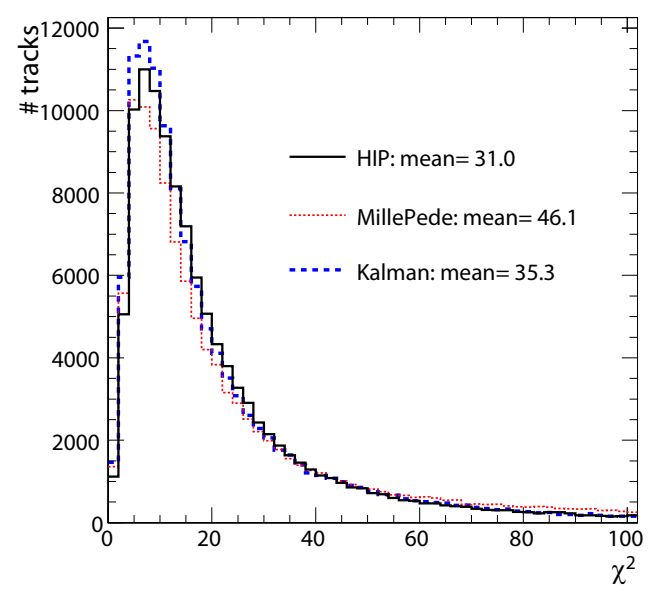

Figure 2: Comparison of absolute $\chi^{2}$-values of the track fits for the track based alignment algorithms (reference subset of modules).

The corrections $\Delta \phi$ for the TEC disks obtained independently with the laser alignment system and with the track based Kalman filter algorithm are displayed in Figure 3. The uncertainties on the LAS results are calculated from the spread of the individual measurements with the four active laser beams. This spread is interpreted as misalignment on module and petal level to which the LAS is insensitive. Within the uncertainties, the estimated corrections are in good agreement with the track based results.

\section{Monte Carlo results}

During spring and summer of 2008, the Computing, Software and Analysis challenge (CSA08) aimed to test the full scope of data handling, analysis activities and alignment work flow needed for LHC data taking in 2008 with Monte Carlo samples. All three track based alignment algorithms were used in this procedure. For two early LHC operation scenarios, the amount of data according to the number of events available within approximately six days was simulated: 


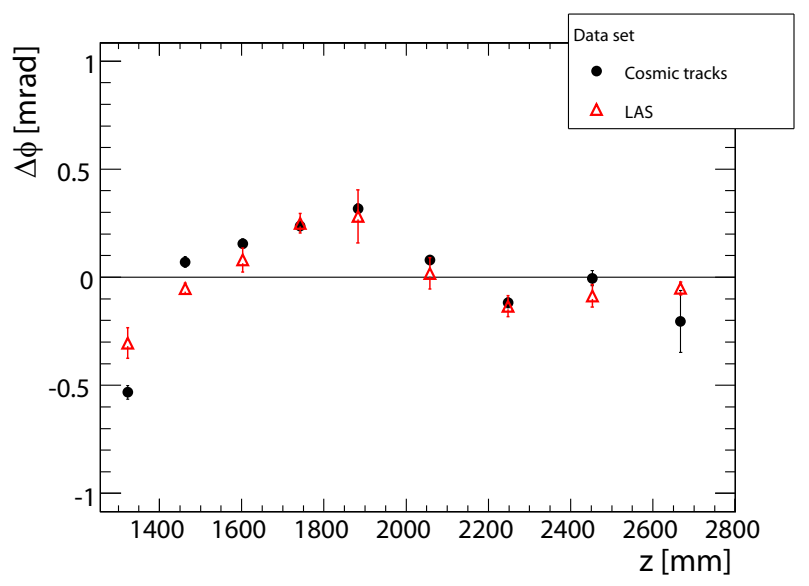

Figure 3: Comparison of $\Delta \phi$ corrections for the nine TEC disks obtained with track based alignment and with the LAS.

- the $S 43$ scenario for operation with 43 bunches per beam at a luminosity of $\mathcal{L}=2 \cdot 10^{30} \mathrm{~cm}^{-2} \mathrm{~s}^{-1}$, resulting in about $1 \mathrm{pb}^{-1}$ of data, and

- the $S 156$ scenario simulating 156 bunches per beam at $\mathcal{L}=2 \cdot 10^{31} \mathrm{~cm}^{-2} \mathrm{~s}^{-1}$, delivering about $10 \mathrm{pb}^{-1}$.

Misalignment has been applied to all simulated data according to the expected remaining uncertainties after survey data, LAS measurements and track based alignment with cosmic muons have been employed.

The simulated data comprise a total of 9 million minimum bias events with track $p_{T}$ above $1.5 \mathrm{GeV} / c$ and 1.3 million single isolated muons which were partly filtered from di-jet events. In addition, for the S156 scenario, 10 million cosmic muons and about 1 million muon pairs from $Z$ and $J / \Psi$ decays were simulated.

Using the track based alignment algorithms, knowledge of module positions and orientations can well be improved. Figure 4 shows the distribution of differences between the true values of $r \cdot \Delta \phi$ (being the most sensitive quantity to misalignment) and those obtained from the alignment procedure with the MillePede algorithm. Additionally, the distribution for the estimated initial conditions at startup is given. Using the track based alignment results from the S43 and S156 Monte Carlo samples, the resolution along this coordinate can be improved to $37 \mu \mathrm{m}$ and $35 \mu \mathrm{m}$, respectively.

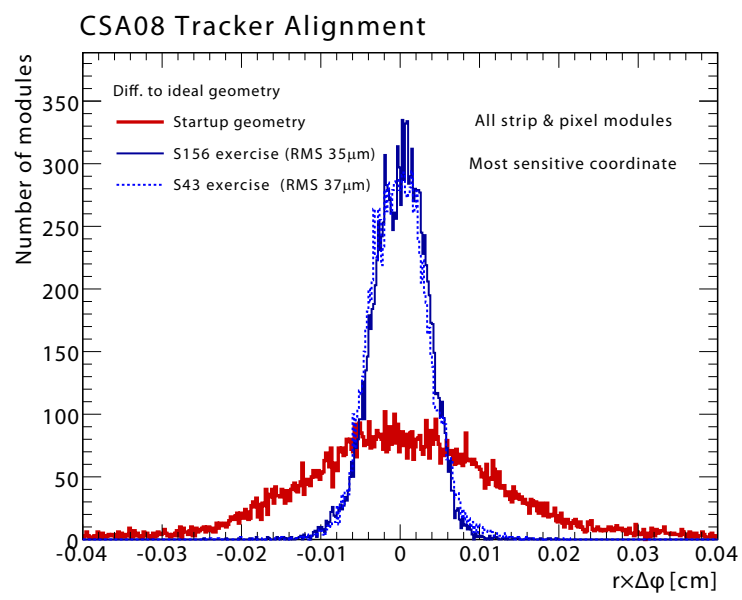

Figure 4: Distribution of differences between true and determined values of $r \cdot \Delta \phi$ for initial conditions (startup) and after alignment with the MillePede algorithm for all strip and pixel modules.

The normalised track $\chi^{2}$ distributions after alignment as well as based on the ideal and the initial geometry are displayed in Figure 5. Compared to the latter, whose lowermost tail only is visible at the very bottom of the panel, the distributions obtained after alignment show distinct peaks close to $\chi^{2} \approx 1$. The distribution for the $\mathrm{S} 43$ scenario has a mean of $\left\langle\chi^{2}\right\rangle=1.40$ while that for $\mathrm{S} 156$ gives $\left\langle\chi^{2}\right\rangle=1.35$ (ideal geometry: $\left\langle\chi^{2}\right\rangle=1.34$ ). The algorithm 
performs well in minimizing the chi-square function equation (1).

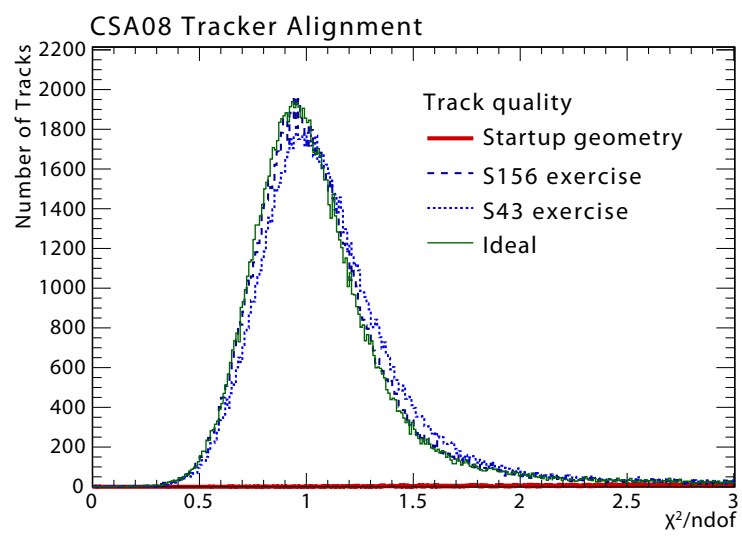

Figure 5: $\chi^{2}$ distributions from track reconstruction using the MillePede alignment results, and based on ideal and initial (startup) geometries. Of the latter only the lower tail is visible at the very bottom of the panel.

The impact of the alignment procedure on the tracker's physics performance can be seen from Figure 6 which gives the distributions of measured $p_{T}$ for $100 \mathrm{GeV} / c$ simulated muons reconstructed using the alignment results and based on ideal as well as initial conditions. Compared to the latter, there is a significant improvement of the $p_{T}$ resolution (as the width of a Gaussian fit) to $\sigma_{p_{T}}=3.0 \mathrm{GeV} / c$ for the $\mathrm{S} 43$ scenario which updates to even $\sigma_{p_{T}}=2.2 \mathrm{GeV} / c$ in case $\mathrm{S} 156$ samples are used. This improvement is a benefit from additionally deploying cosmic muon and resonance decay data in combination with further constraints. With the ideal geometry, one obtains $\sigma_{p_{T}}=1.7 \mathrm{GeV} / c$.

Consequently, even with close to ideal track $\chi^{2}$ distributions, differences with respect to the ideal geometry can be observed. This is interpreted as the result of $\chi^{2}$-invariant deformations of the tracker, not aligned degrees of freedom, or outliers. Given the improvement observed with S156 samples, the remaining misalignment can further be reduced using more data and additional data samples such as from beam halo muons.

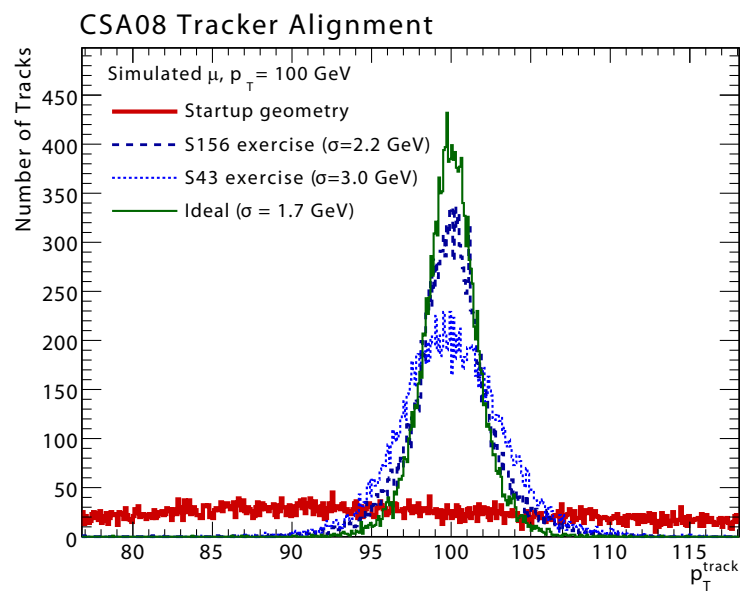

Figure 6: Distributions of measured $p_{T}$ for $100 \mathrm{GeV} / \mathrm{c}$ muons reconstructed using the MillePede alignment results, and based on ideal and initial (startup) geometries.

\section{Conclusions}

Aligning all CMS silicon tracker modules to a precision of a few dozen micrometres is a highly complex task and relies on data from survey measurements, the hardware based laser alignment system and redundant algorithms for track based alignment. With first cosmic muon data it has been shown that the overall strategy significantly improves track quality. Using Monte Carlo samples reflecting the data available at a very early stage of LHC operation proves the good performance of the track based alignment. 


\section{References}

[1] CMS Collaboration, J. Instrum. 3 (2008) S08004

[2] A.Ostaptchouk et al., CMS NOTE-2001/53 (2001)

[3] V. Karimaki, T. Lampen, F.-P. Schilling, CMS NOTE-2006/018 (2006)

[4] E. Widl, R. Frühwirth, W. Adam, CMS NOTE-2006/022 (2006)

[5] V. Blobel, Nucl. Instrum. Meth. A 566 (2006) 5 